\title{
TOURISM PROVIDERS, ACHIEVEMENT, CULTURE AND JOB SATISFACTION IN SERBIA
}

\author{
Jelena Vukonjanski Srdić ${ }^{1}$ (D) \\ Bojana Ostojić ${ }^{2}$
}

DOI: https://doi.org/10.31410/tmt.2020.485

\begin{abstract}
This paper aims to investigate the relationships between GLOBE national culture dimensions, the facets of job satisfaction and the need for achievement relation in Serbian tourism providers. The sample consists of 120 managers in the Serbian service sector/tourism providers in Serbia (transportation, utility companies, telecommunications, and banks). The results obtained proved that national culture dimensions are significant predictors of all facets of job satisfaction and that the relations between some organizational culture dimensions and the facets of job satisfaction are moderated by the need for achievement variable. Our results may be of interest to human resources managers in the Serbian tourism service providers.
\end{abstract}

Keywords: National culture, Job satisfaction, Need for achievement, Gender, Tourism providers, Serbia.

\section{INTRODUCTION}

$\mathrm{T}$ he last two decades were the time of political and economic changes in Serbia and many Eastern European countries. Transitional societies have significant cultural differences that are based on different histories and transitional pathways (Mebrahtu et al., 2000). Joint political programs, the power, and the mobility of transnational capital, as well as the impact on the individual national system, are comprehensive evidence that social systems fundamentally converge in terms of the functioning of the regulatory system, and the knowledge from the organizational level clearly indicates that national differences are what moderate the factors that affect the way organizations work (Blyton, 2001). Feichtinger and Fink (1998) argue that cultural and similar processes in transition countries that have a communist legacy produce a ,cultural shock”. In transition countries, culture shock is the product of complex economic, social, and political forces, and an important conceptual tool for managers who are responsible for international strategy in transition countries, as established by Fink and Holden (2002). Managers must be aware of the cultural differences and similarities in order to be successful in working with people from other cultures (Javidan, House, 2001; Gerhart, 2008), this is of particular importance to the service sector.

Globalization has also affected changes in the service sector. Due to the changes, but also due to the development of tourism, an important factor in the quality of the tourist destination is tourism providers. On the other hand, according to Kirin \& Gavrić (2017), the main problems of the tourism sector in Serbia relate to tourism providers (e.g. road infrastructure, renovation of facades, traffic jams, etc.) which require large human and material resources. Zouni \& Kouremenos (2008) emphasize the importance of effective inclusion of local factors (main destination stakeholders) in the successful operation, sustainability, and long-term viability of a tourist destination. The authors indicate that destination competitiveness can be improved by coordi-

\footnotetext{
University Educons, Project Management College, Bože Jankovića 14, Belgrade, Serbia
}

University Educons, Project Management College, Bože Jankovića 14, Belgrade, Serbia 
nation of evaluations between the most directly involved parts of destination, tourists (tourism demand), and tourism professionals (tourism supply). The research regarding resident attitudes to tourism development shows positive results towards the provision of the community facility, job creation, and the promotion of the area for tourism (Mason, Cheyne, 2000).

This paper aims to investigate the relationships between GLOBE national culture dimensions, the facets of job satisfaction and the need for achievement and gender in tourism service providers in Serbia (transportation, utility companies, telecommunications, and banks).

Hofstede (1980) has investigated cultural dimensions in some Yugoslav republics (Slovenia, Croatia and Serbia). After the break-up of Yugoslavia, it was determined that in Serbia power distance (86) and uncertainty avoidance (92) indexes were very high, and individualism (25) and masculinity indexes (43) were very low (Hofstede, 2001, p 501). Hofstede (2010) stated that all organizations are embedded within societal cultures, which are likely to have an ambient influence on the organizations embedded within them. GLOBE project accepts Hofstede's model and paradigm but also presents an important point in the development of Hofstede's doctrine.

According to Morris et al. (1994), individualism/collectivism is an important factor in understanding entrepreneurial behavior in the organization, but it can be assumed that corporate entrepreneurship depends more on collective actions and cooperative spirit than on independent/ individualistic actions (Finkelstein, 2011). The findings from Tiessen (1997) show that individualism and collectivism neither encourage nor discourage entrepreneurship, they influence how its functions are accomplished - individualists produce breakthroughs that collectivists implement and improve. He suggests the best entrepreneurs need to connect with others and with the help of others make their ideas better and achieve their goals. It can be assumed that the same refers to intrapreneurs. National cultures are especially important for intrapreneurship in the tourism industry, particularly for tourism providers, in order to maximize the quality of the tourist destination. Wakkee et al. (2010) point out that in the long run entrepreneurial behavior within existing organizations (intrapreneurship) has a positive effect on the financial performance of organizations, and that the creation of entrepreneurial employees is important in traditional service sectors. Intrapreneurship can be developed in firms of different sizes, including tourism and trade and it should be promoted as the key for business success (Auer Antoncic $\&$ Antoncic, 2018). Due to all the above facts, we investigate national culture as a predictor of job satisfaction and moderating effect of need for achievement on the relation between national culture (as an independent variable) and job satisfaction (as a dependent variable) in the tourism service providers in Serbia.

\section{NATIONAL CULTURE, JOB SATISFACTION AND NEED FOR ACHIEVEMENT}

\subsection{National culture}

Among the first researchers who recognized the importance of national culture and its impact on organizations were Kluckhohn and Strodtbeck (1961). Besides them, numerous other researchers were dedicated to defining the cultural dimensions and their influence on the organization (Hall, 1976; Triandis, 1995; Hofstede, 1980, 2001; Trompenaars \& Hampden-Turner, 1997; House et al., 2004; Dimitratos et al., 2011; Gerhart, 2008; Dickson et al., 2003, etc.). One of the reasons why national culture is important is the assumption that it is a constraint on man- 
agement practices and organizational culture (Gerhart, 2008). Besides, culture is an important factor that determines the profile of individual values, but also the values of the organizational culture of the companies operating within it and significantly affects the organizational culture (Hofstede 1980, 2001; Trompenaars \& Hampden-Turner, 1997). National culture can be a significant variable that influences the development of cognitive style and perception of managers (Dimitratos et al., 2011). Dickson et al. (2003) point out that the GLOBE dimensions of culture (Javidan \& House, 2001) serve the needs of global managers to have the cultural ability or to be sensitive to cultural differences.

Knowing the cultural dimensions and the characteristics of different cultures can help managers in tourism and organizations from other sectors to create a culture that contributes to employees being motivated and satisfied (Paunković \& Jovanović 2016). Researchers emphasize the importance of culture in the service sector. Nazarian et al. (2017) examine the impact of national culture on the hotel industry. The results obtained show that culture is an important factor for achieving a balanced organizational culture and that has an influence on performance. Studies conducted by Hui et al. (2004) in hotel employees show that power distance has a moderate effect on the relationship between empowerment and job satisfaction. Merritt (2000) indicates that national culture has a greater influence than the professional culture of airline pilots. Alajmi et al. (2011) found that national culture dimensions, power distance and uncertainty avoidance affect the information flow and communication policy in the service sector. Pavluković et al. (2017) examined the impact of national culture on local residents' attitudes towards tourism. Based on previous studies, in the service sector in Serbia, culture dimensions are predictors of the dimensions of entrepreneurial orientation (Nedeljković Knežević \& Pavluković, 2020).

For this study, we used the GLOBE model. The initial aim of the GLOBE (Global Leadership and Organizational Behavior) research project was the development of national and organizational measures of culture and leadership applicable to cross-cultural research. The GLOBE cultural dimensions are based on several studies (Hofstede, 1980; 2001; House et al., 1999; Dickson et al. 2000). The GLOBE project has empirically established nine cultural dimensions (for national and organizational culture) that were covered by 78 survey questions, half of them asking subjects to describe their culture ('as is') and the other half refers to what should be ('should be'). GLOBE is a multi-phase, multi-method, and the nine "as is" GLOBE cultural dimensions are (House et al., 2002; House et al., 2004; Vukonjanski et al., 2012):

1. Uncertainty Avoidance: The extent to which a society, organization, or group relies on social norms, rules, and procedures to alleviate unpredictability of future events.

2. Future Orientation: The degree to which a collective encourages and rewards future-oriented behaviors such as planning and delaying gratification.

3. Power Distance: The degree to which the members of a collective expect power to be distributed equally.

4. Collectivism I (Institutional): The degree to which organizational and societal institutional practices encourage and reward the collective distribution of resources and collective action.

5. Humane Orientation: The degree to which a collective encourages and rewards individuals for being fair, altruistic, generous, caring, and kind to others.

6. Performance Orientation: The degree to which a collective encourages and rewards group members for performance improvement.

7. Collectivism II (In-Group): The degree to which individuals express pride, loyalty, and cohesiveness in their organizations or families. 
8. Gender Egalitarianism: The degree to which a collective minimizes gender inequality.

9. Assertiveness: The degree to which individuals are assertive, confrontational, and aggressive in their relationships with others.

\subsection{Job satisfaction}

There are many definitions of job satisfaction. Job satisfaction is a pleasurable or positive emotional state resulting from the appraisal of one's job or job experiences (Locke, 1976). Also, job satisfaction is an attitude reflecting how well people like or dislike their job, a person's evaluation of his/her job, and work context (Spector, 1997). Job satisfaction is viewed as an attitude to the job in terms of global satisfaction or terms of facet of satisfaction including the pay, work itself, supervision, promotion opportunities, benefits, rewards, procedures, communication and coworkers. In this paper, Spector's definition will be applied.

According to Spector (1997), there are three reasons for the evaluation of the employees' job satisfaction. First, employees should be respected as individuals that could contribute to general organizational success. Second, evaluation of the employees' job satisfaction could serve as an indicator of the business effectiveness among the employees. Third, the job satisfaction of the employees might cause positive effects on the general fulfillment of the organizational goals. Job satisfaction is linked to the motivation, productivity, efficiency, and work-related satisfaction of employees (Hwang \& Chi, 2005). On the other hand, job dissatisfaction can significantly affect the behavior of employees resulting in absence from work, complaints and grievances, and termination of employment (Sempane et al., 2002). To create value for companies and society, employers need to generate job satisfaction because of its importance at the individual, organizational, and societal levels, including employees' perception of their job satisfaction (Díaz-Carrión et al. 2020).

Job satisfaction is one of the predictors of various significant organizational outcomes, including job performance (Judge et al., 2001). One of the most significant research in this area (Judge et al., 2001), has led to a series of conclusions about the relationship and the influences between job satisfaction and productivity. This research shows that the greatest impact has factors related to organizational culture and personal determinants. Hwang \& Chi (2005) findings show significant correlations between employee job satisfaction, internal marketing and performance of international hotels. Fargher et al. (2008) investigated the relationship between culture and job satisfaction in Eastern and Western European countries. The study points to the strong influence of national culture on an individual's attitude towards job satisfaction, and significant differences between employees in Eastern and Western Europe are observed, which are primarily caused by the importance of family and religion. Sempane et al., (2002) establish a significant relationship between the variables of job satisfaction and the organizational culture of employees within a service organization. Job satisfaction in the tourist sector in Europe varies based on the model of working conditions. (Díaz-Carrión et al. 2020). Job satisfaction in service industries varies in different countries, occupations, employment contracts and earnings levels (Bednarska \& Szczyt, 2015). The same authors summarized that gender, tenure, age and sector do not affect job satisfaction.

\subsection{Need for Achievement}

The need for achievement refers to a person's responsibility for involvement in activities to achieve the desired outcome, and it is associated with a positive mood, task interest, and organizational spontaneity (Eisenberger et al., 2005). Finogenow (2017) defines the need for achieve- 
ment as a consistent concern for doing things better and a desire to accomplish excellent results in conditions of high expectations. High achievement individuals are highly motivated by competing and challenging work and they look for promotional opportunities and have a strong urge for feedback on their achievement (McClelland, 1961). High need for achievement employees base on their self-esteem, successful development and the utilization of their skills and talents (Eisenberger et al., 2005: McClelland,1961). In case of low need for achievement, employees are less interested in the activities that provide high skill and challenge at work and try to avoid competence assessment (Eisenberger et al., 2005).

In recent years the need for achievement has gained importance in the study of entrepreneurship. Based on the research that highlights the importance of matching the need for achievement and business goals, the need for achievement is positively related to entrepreneurial persistence and business goals are moderators on the relationship between the need for achievement and persistence (Wu et al., 2007). Auer Antoncic \& Antoncic (2018) indicate positive relationships between the need for achievement of the female entrepreneur and intrapreneurship, and between intrapreneurship and firm growth.

Within this study we hypothesize:

H1: National culture dimensions significantly predict facets of the job satisfaction.

H2: The need for achievement moderates the regression between national culture dimension and the facet of job satisfaction.

H3: Gender moderates the regression between national culture dimension and the facet of job satisfaction.

\section{METHOD}

\subsection{Participants and data collection}

The research was carried out in organizations in Serbia (Vojvodina, Belgrade, Sumadija, Southern, Eastern, and Western Serbia). The survey was conducted by the respondents completing the questionnaire by using the standard pen-and-paper procedure. The sample consists of 120 managers from the service sector in Serbia (transportation, utility companies, telecommunications, banks). 65 of the respondents are male and 55 are female. According to respondents' age levels, results are presented in Table 1.

Table 1. Respondents age levels

\begin{tabular}{|c|c|c|c|}
\hline Age & $\%$ & Male & Female \\
\hline$<29$ & $17 \%$ & $60 \%$ & $40 \%$ \\
\hline $\mathbf{3 0 - 3 9}$ & $33 \%$ & $67 \%$ & $33 \%$ \\
\hline $\mathbf{4 0 - 5 4}$ & $40 \%$ & $47 \%$ & $52 \%$ \\
\hline $\mathbf{5 9}>$ & $11 \%$ & $31 \%$ & $69 \%$ \\
\hline
\end{tabular}

Source: Authors calculation

This sample is part of a wider study that included 340 respondents (managers) from different sectors - Industrial, Trade, Service sector, Public administration, Agriculture (demographic question). Collected data were analyzed by using the Statistical Program for Social Sciences. Statistical analyses were primarily focused on determining the moderator's effects of the Need for Achievement on the correlation between the national culture dimensions and job satisfaction, as the main constructs of the research. 


\subsection{Survey instruments}

Standard instruments are used to measure national culture, job satisfaction and need for achievement.

We used the GLOBE project instrument for measuring the national culture dimension "as is" since the GLOBE project presents an additional, expanded model of cultural measures (Venaik/ Brewer 2008). The "should be" part of the instrument is used only in order to compare the values obtained from this part of the GLOBE instrument with the values from the "as is" part of the instrument. The GLOBE questionnaire has 34 items and the answers are measured on the 7- point Likert scale. These instruments measure nine organizational culture dimensions: Uncertainty Avoidance, Future Orientation, Power Distance, Collectivism 1, Humane Orientation, Performance Orientation, Collectivism 2, Gender Egalitarianism and Assertiveness (House et al. 1999, 2002, 2004).

Job satisfaction is measured by the Job Satisfaction Survey (Spector 1985). The questionnaire has 36 items related to nine facets of job satisfaction, and answers are measured by a 6-point Likert scale. Dimensions of job satisfaction are defined and described in the following way (Spector, 1985; Vukonjanski et al., 2012): JS1- Pay (Pay and remuneration), JS2 - Promotion (Promotion opportunities), JS3 - Supervision (Immediate supervisor), JS4 - Fringe Benefits (Monetary and nonmonetary fringe benefits), JS5 - Contingent Rewards (Appreciation, recognition, and rewards for good work), JS6 - Operating Procedures (Operating policies and procedures), JS7 - Co-workers (People you work with), JS8 - Nature of Work (Job tasks themselves), JS9 - Communication (Communication within the organization).

We used the Need for Achievement Questionnaire (Wu et al., 2007), measured by a 5-point Likert scale. Need for Achievement instrument measures: need to face challenges, need to learn constantly, need for personal growth and need to succeed.

\section{RESEARCH RESULTS}

The descriptive statistics for the items and the dimensions of the observed questionnaires: "as is" dimensions of the national culture ( $\mathrm{NCi}, \mathrm{i}=1,2,3,4,5,6,7,8,9)$, the facet of job satisfaction ( $\mathrm{JSj}$, $\mathrm{j}=1,2,3,4,5,6,7,8,9)$ and Need for Achievement (NA) are shown in Table 2. The table shows the names of all dimensions, abbreviation for each dimension that we shall use later in the paper, mean, standard deviation of all the items and dimensions, as well as Cronbach's alpha for each dimension. The Cronbach's alpha values vary in the range from a $\alpha=0.606$ to $\alpha=0.874$.

Table 2. Descriptive Statistics

\begin{tabular}{|l|l|l|l|l|l|l|l|}
\cline { 2 - 8 } \multicolumn{1}{c|}{} & Abbrev. & N & Min. & Max. & Mean & $\begin{array}{l}\text { Std. } \\
\text { Deviation }\end{array}$ & $\boldsymbol{\alpha}$ \\
\hline Uncertainty Avoidance & NC1 & 120 & 1.00 & 6.75 & 3.1437 & 1.29708 & .723 \\
\hline Future Orientation & NC2 & 120 & 1.00 & 6.40 & 2.2767 & 1.02414 & .771 \\
\hline Power Distance & NC3 & 120 & 3.40 & 7.00 & 6.1617 & .83748 & .725 \\
\hline Collectivism 1 & NC4 & 120 & 1.00 & 6.25 & 3.6375 & 1.06602 & .699 \\
\hline Humane Orientation & NC5 & 120 & 1.00 & 6.80 & 3.2867 & 1.27411 & .801 \\
\hline Performance Orientation & NC6 & 120 & 1.00 & 6.67 & 3.0694 & 1.31066 & .736 \\
\hline Collectivism 2 & NC7 & 120 & 2.50 & 7.00 & 5.2271 & .92070 & .653 \\
\hline Gender Egalitarianism & NC8 & 120 & 1.60 & 5.20 & 3.3667 & .78701 & .655 \\
\hline Assertiveness & NC9 & 120 & 2.00 & 7.00 & 4.0500 & .81610 & .606 \\
\hline
\end{tabular}




\begin{tabular}{|l|l|l|l|l|l|l|l|}
\hline Pay & JS1 & 120 & 1.00 & 6.00 & 2.9187 & 1.28467 & .788 \\
\hline Promotion & JS2 & 120 & 1.00 & 6.00 & 3.3583 & 1.17338 & .680 \\
\hline Supervision & JS3 & 120 & 1.00 & 6.00 & 3.5583 & 1.31520 & .746 \\
\hline Fringe Benefits & JS4 & 120 & 1.00 & 5.50 & 2.8667 & 1.17972 & .736 \\
\hline Contingent Rewards & JS5 & 120 & 1.00 & 6.00 & 2.9979 & 1.31671 & .780 \\
\hline Operating Procedures & JS6 & 120 & 1.00 & 6.00 & 3.2646 & .94062 & .649 \\
\hline Coworkers & JS7 & 120 & 1.00 & 6.00 & 4.3250 & 1.13417 & .767 \\
\hline Nature of Work & JS8 & 120 & 1.00 & 6.00 & 4.4542 & 1.26979 & .834 \\
\hline Communication & JS9 & 120 & 1.00 & 6.00 & 3.8000 & 1.30634 & .764 \\
\hline Need for Achievement & NA & 120 & 1.00 & 5.00 & 4.0896 & .90505 & .874 \\
\hline
\end{tabular}

Source: Authors calculation

The national cultural dimension NC3 (Power Distance) has the highest value (6.16), followed by NC7 (Collectivism 2, 5.22), and the lowest value has NC2 (Future Orientation, 2.27). In terms of job satisfaction, the highest value has dimension JS8 (Nature of Work, 4.45), followed by JS7 (Coworkers, 4.32), and Dimension JS1 (Pay, 2.9) has the lowest value.

In Table 3 we give the average values of the "as is" and the "should be" dimensions of national culture in Serbia (tourism providers) and for the overall GLOBE Data (Javidan \& House 2001).

Table 3. National culture dimensions

\begin{tabular}{|l|c|c|c|c|c|c|}
\cline { 2 - 6 } \multicolumn{1}{c|}{} & \multicolumn{2}{|c|}{ Tourism providers } & \multicolumn{4}{|c|}{$\begin{array}{c}\text { Overall GLOBE Data } \\
\text { (Javidan \& House, 2001) }\end{array}$} \\
\cline { 3 - 7 } & \multicolumn{2}{|c|}{} & \multicolumn{2}{c|}{ As Is } & \multicolumn{2}{c|}{ Should Be } \\
\cline { 2 - 7 } & \multirow{2}{*}{ As Is } & Should Be & $\begin{array}{c}\text { Highest } \\
\text { score }\end{array}$ & $\begin{array}{c}\text { Lowest } \\
\text { Score }\end{array}$ & $\begin{array}{c}\text { Highest } \\
\text { score }\end{array}$ & $\begin{array}{c}\text { Lowest } \\
\text { Score }\end{array}$ \\
\hline Uncertainty Avoidance & 3.14 & 5.60 & 5.37 & 2.88 & 5.61 & 3.16 \\
\hline Future Oriented & 2.28 & 6.23 & 5.07 & 2.88 & 6.20 & 2.95 \\
\hline Power Distance & 6.16 & 2.98 & 5.80 & 3.89 & 4.35 & 2.04 \\
\hline Collectivism 1 & 3.64 & 4.88 & 5.22 & 3.25 & 5.65 & 3.83 \\
\hline Humane Orientation & 3.29 & 6.28 & 5.12 & 3.18 & 6.09 & 3.39 \\
\hline Performance Orientation & 3.07 & 6.52 & 4.90 & 2.88 & 6.58 & 2.35 \\
\hline Collectivism 2 & 5.22 & 5.75 & 6.03 & 3.53 & 6.52 & 4.06 \\
\hline Gender Egalitarianism & 3.37 & 4.97 & 4.08 & 2.50 & 5.17 & 3.18 \\
\hline Assertiveness & 4.05 & 2.72 & 4.73 & 3.38 & 5.56 & 2.66 \\
\hline
\end{tabular}

Source: Authors calculation

Table 4 presents gender differences in perception of national culture and job satisfaction of tourism providers in Serbia.

Table 4. Gender differences

\begin{tabular}{|l|l|l|l|l|l|}
\hline National culture & Male & Female & Job satisfaction & Male & Female \\
\hline Uncertainty Avoidance & 3.23 & 3.04 & Pay & 3.27 & 2.50 \\
\hline Future Oriented & 2.41 & 2.12 & Promotion & 3.54 & 3.15 \\
\hline Power Distance & 6.14 & 6.19 & Supervision & 3.76 & 3.32 \\
\hline Collectivism 1 & 3.77 & 3.48 & Fringe Benefits & 3.07 & 2.62 \\
\hline Humane Orientation & 3.46 & 3.08 & Contingent Rewards & 3.38 & 2.55 \\
\hline Performance Orientation & 3.39 & 2.69 & Operating Procedures & 3.34 & 3.17 \\
\hline Collectivism 2 & 5.23 & 5.22 & Coworkers & 4.46 & 4.16 \\
\hline Gender Egalitarianism & 3.28 & 3.47 & Nature of Work & 4.60 & 4.28 \\
\hline Assertiveness & 3.99 & 4.12 & Communication & 3.86 & 3.73 \\
\hline
\end{tabular}

Source: Authors calculation 
In Table 5 we give the Pearson coefficients of the correlation between national culture dimensions and the facet of job satisfaction.

Table 5. Pearson coefficients of the correlation between national culture and job satisfaction

\begin{tabular}{|l|l|l|l|l|l|l|l|l|l|}
\cline { 2 - 9 } \multicolumn{1}{c|}{} & NC1 & NC2 & NC3 & NC4 & NC5 & NC6 & NC7 & NC8 & NC9 \\
\hline JS1 & $.383^{* *}$ & $.404^{* *}$ & $-.390^{* *}$ & $.289^{* *}$ & $.345^{* *}$ & $.525^{* *}$ & .117 & -.033 & -.035 \\
\hline JS2 & $.198^{*}$ & $.301^{* *}$ & $-.328^{* *}$ & $.373^{* *}$ & $.299^{* *}$ & $.327^{* *}$ & .129 & .045 & .024 \\
\hline JS3 & $.308^{* *}$ & $.379^{* *}$ & $-.413^{* *}$ & $.203^{*}$ & $.354^{* *}$ & $.343^{* *}$ & .160 & .071 & -.033 \\
\hline JS4 & $.404^{* *}$ & $.385^{* *}$ & $-.262^{* *}$ & $.202^{*}$ & $.299^{* *}$ & $.414^{* *}$ & $.205^{*}$ & -.113 & .120 \\
\hline JS5 & $.370^{* *}$ & $.326^{* *}$ & $-.361^{* *}$ & $.325^{* *}$ & $.433^{* *}$ & $.439^{* *}$ & .141 & .132 & .055 \\
\hline JS6 & -.011 & -.039 & -.036 & .125 & .084 & .035 & -.149 & .079 & -.126 \\
\hline JS7 & $.230^{*}$ & $.238^{* *}$ & $-.191^{*}$ & $.235^{* *}$ & $.282^{* *}$ & $.248^{* *}$ & $.200^{*}$ & .159 & .000 \\
\hline JS8 & $.185^{*}$ & $.213^{*}$ & -.089 & .070 & .116 & $.212^{*}$ & $.231^{*}$ & .135 & .080 \\
\hline JS9 & $.341^{* *}$ & $.267^{* *}$ & $-.364^{* *}$ & $.189^{*}$ & $.321^{* *}$ & $.395^{* *}$ & $.369^{* *}$ & .036 & .057 \\
\hline
\end{tabular}

** Correlation is significant at the 0.01 level (2-tailed).

* Correlation is significant at the 0.05 level (2-tailed).

Source: Authors calculation

By the multiple regression method, we also investigated the contribution of all the independent variables of national culture dimensions to the total R-square in the regression with the facet of job satisfaction as a dependent variable. The dimensions of the national culture are the best predictor for Pay $\left(\mathrm{R}^{2}=0.336\right)$. This tells us that $34 \%$ of the variation in pay can be predicted using GLOBE dimensions of national culture. National culture is not a predictor of JS6 (Operating Procedures) and JS8 (Nature of Work). Multiple regression analysis is presented in Table 6 and Figure 1.

Table 6. Multiple regression analysis

\begin{tabular}{|c|c|c|c|c|c|c|c|}
\hline Dependent & Independent & $\beta$ & $\mathrm{t}$ & Sig. & $\mathbf{R}^{2}$ & $\mathbf{F}$ & Sig. \\
\hline \multirow{9}{*}{ JS1 } & $\mathrm{NC} 1$ & .071 & .654 & .514 & \multirow{9}{*}{.336} & \multirow{9}{*}{6.198} & \multirow{9}{*}{.000} \\
\hline & $\mathrm{NC} 2$ & .102 & .951 & .344 & & & \\
\hline & NC3 & -.132 & -1.182 & .240 & & & \\
\hline & NC4 & .067 & .751 & .454 & & & \\
\hline & NC5 & .021 & .207 & .837 & & & \\
\hline & \multirow{4}{*}{$\begin{array}{l}\text { NC6 } \\
\text { NC7 } \\
\text { NC8 } \\
\text { NC9 }\end{array}$} & .337 & 2.879 & .005 & & & \\
\hline & & .038 & .465 & .643 & & & \\
\hline & & -.105 & -1.276 & .205 & & & \\
\hline & & -.158 & -1.964 & .052 & & & \\
\hline \multirow{9}{*}{ JS2 } & $\mathrm{NC1}$ & -.160 & -1.369 & .174 & \multirow{9}{*}{.238} & \multirow{9}{*}{3.822} & \multirow{9}{*}{.000} \\
\hline & $\mathrm{NC} 2$ & .064 & .557 & .578 & & & \\
\hline & NC3 & -.261 & -2.177 & .032 & & & \\
\hline & $\mathrm{NC4}$ & .283 & 2.952 & .004 & & & \\
\hline & NC5 & .086 & .798 & .427 & & & \\
\hline & NC6 & .058 & .462 & .645 & & & \\
\hline & NC7 & .129 & 1.461 & .147 & & & \\
\hline & NC8 & -.020 & -.225 & .822 & & & \\
\hline & NC9 & -.042 & -.484 & .629 & & & \\
\hline \multirow{9}{*}{ JS3 } & $\mathrm{NC1}$ & .000 & -.002 & .998 & \multirow{9}{*}{.270} & \multirow{9}{*}{4.521} & \multirow{9}{*}{.000} \\
\hline & $\mathrm{NC} 2$ & .176 & 1.570 & .119 & & & \\
\hline & $\mathrm{NC} 3$ & -.333 & -2.842 & .005 & & & \\
\hline & $\mathrm{NC} 4$ & .013 & .141 & .888 & & & \\
\hline & NC5 & .129 & 1.213 & .228 & & & \\
\hline & NC6 & -.038 & -.309 & .758 & & & \\
\hline & NC7 & .174 & 2.011 & .047 & & & \\
\hline & NC8 & -.027 & -.316 & .753 & & & \\
\hline & NC9 & -.135 & -1.607 & .111 & & & \\
\hline
\end{tabular}




\begin{tabular}{|c|c|c|c|c|c|c|c|}
\hline \multirow{9}{*}{ JS4 } & $\mathrm{NC1}$ & .192 & 1.679 & .096 & \multirow{9}{*}{.267} & \multirow{9}{*}{4.453} & \multirow{9}{*}{.000} \\
\hline & \multirow{8}{*}{$\begin{array}{l}\mathrm{NC} 2 \\
\mathrm{NC} 3 \\
\mathrm{NC} 4 \\
\mathrm{NC} 5 \\
\mathrm{NC} 6 \\
\mathrm{NC} 7 \\
\mathrm{NC} 8 \\
\mathrm{NC} 9 \\
\end{array}$} & .159 & 1.419 & .159 & & & \\
\hline & & -.016 & -.134 & .893 & & & \\
\hline & & -.031 & -.327 & .744 & & & \\
\hline & & .040 & .375 & .708 & & & \\
\hline & & .182 & 1.481 & .141 & & & \\
\hline & & .114 & 1.319 & .190 & & & \\
\hline & & -.158 & -1.835 & .069 & & & \\
\hline & & .011 & .136 & .892 & & & \\
\hline \multirow{9}{*}{ JS5 } & \multirow{9}{*}{$\begin{array}{l}\mathrm{NC} 1 \\
\mathrm{NC} 2 \\
\mathrm{NC} 3 \\
\mathrm{NC} 4 \\
\mathrm{NC} 5 \\
\mathrm{NC} 6 \\
\mathrm{NC} 7 \\
\mathrm{NC} 8 \\
\mathrm{NC} 9 \\
\end{array}$} & .064 & .564 & .574 & \multirow{9}{*}{.287} & \multirow{9}{*}{4.919} & \multirow{9}{*}{.000} \\
\hline & & -.045 & -.406 & .686 & & & \\
\hline & & -.091 & -.783 & .436 & & & \\
\hline & & .140 & 1.504 & .136 & & & \\
\hline & & .215 & 2.048 & .043 & & & \\
\hline & & .193 & 1.589 & .115 & & & \\
\hline & & .107 & 1.252 & .213 & & & \\
\hline & & .076 & .896 & .372 & & & \\
\hline & & -.047 & -.568 & .571 & & & \\
\hline \multirow{9}{*}{ JS6 } & \multirow{9}{*}{$\begin{array}{l}\mathrm{NC} 1 \\
\mathrm{NC} 2 \\
\mathrm{NC} 3 \\
\mathrm{NC} 4 \\
\mathrm{NC5} \\
\mathrm{NC6} \\
\mathrm{NC} 7 \\
\mathrm{NC} 8 \\
\mathrm{NC} 9 \\
\end{array}$} & -.034 & -.264 & .792 & \multirow{9}{*}{.079} & \multirow{9}{*}{1.050} & \multirow{9}{*}{.406} \\
\hline & & -.142 & -1.129 & .261 & & & \\
\hline & & .025 & .190 & .850 & & & \\
\hline & & .157 & 1.491 & .139 & & & \\
\hline & & .095 & .801 & .425 & & & \\
\hline & & .085 & .619 & .537 & & & \\
\hline & & -.149 & -1.536 & .127 & & & \\
\hline & & .066 & .683 & .496 & & & \\
\hline & & -.127 & -1.343 & .182 & & & \\
\hline & $\mathrm{NC1}$ & .025 & .208 & .836 & \multirow{9}{*}{.162} & \multirow{9}{*}{2.363} & \multirow{9}{*}{.018} \\
\hline & $\mathrm{NC} 2$ & .032 & .264 & .792 & & & \\
\hline & NC3 & -.020 & -.156 & .876 & & & \\
\hline & $\mathrm{NC4}$ & .117 & 1.164 & .247 & & & \\
\hline JS7 & NC5 & .151 & 1.332 & .186 & & & \\
\hline & NC6 & .049 & .374 & .709 & & & \\
\hline & $\mathrm{NC7}$ & .195 & 2.102 & .038 & & & \\
\hline & NC8 & .149 & 1.620 & .108 & & & \\
\hline & NC9 & -.065 & -.716 & .475 & & & \\
\hline & $\mathrm{NC} 1$ & .055 & .439 & .661 & & & \\
\hline & $\mathrm{NC} 2$ & .137 & 1.119 & .265 & & & \\
\hline & $\mathrm{NC} 3$ & .105 & .818 & .415 & & & \\
\hline & $\mathrm{NC} 4$ & -.056 & -.548 & .585 & & & \\
\hline JS8 & NC5 & -.024 & -.203 & .840 & .127 & 1.773 & .081 \\
\hline & NC6 & .151 & 1.126 & .263 & & & \\
\hline & NC7 & .201 & 2.133 & .035 & & & \\
\hline & NC8 & .165 & 1.752 & .083 & & & \\
\hline & NC9 & .030 & .326 & .745 & & & \\
\hline & $\mathrm{NC1}$ & .090 & .817 & .416 & & & \\
\hline & $\mathrm{NC} 2$ & -.065 & -.602 & .549 & & & \\
\hline & NC3 & -.289 & -2.559 & .012 & & & \\
\hline & $\mathrm{NC} 4$ & .022 & .248 & .804 & & & \\
\hline JS9 & NC5 & .100 & .980 & .329 & .324 & 5.848 & .000 \\
\hline & NC6 & .087 & .740 & .461 & & & \\
\hline & NC7 & .373 & 4.484 & .000 & & & \\
\hline & NC8 & -.013 & -.157 & .876 & & & \\
\hline & NC9 & -.048 & -.590 & .556 & & & \\
\hline
\end{tabular}

Source: Authors calculation

The variable NC6 (Performance Orientation) has the greatest influence on variable JS1 (Pay). The variable NC4 (Collectivism 1) has the greatest influence on variable JS2 (Promotion). The 
variable NC3 (Power distance) has the greatest influence on variable JS3 (Supervision). The variable NC5 (Humane Orientation) has the greatest influence on variable JS5 (Contingent Rewards). The variable NC7 (Collectivism 2) has the greatest influence on variables JS7 (Coworkers) and JS9 (Communication).

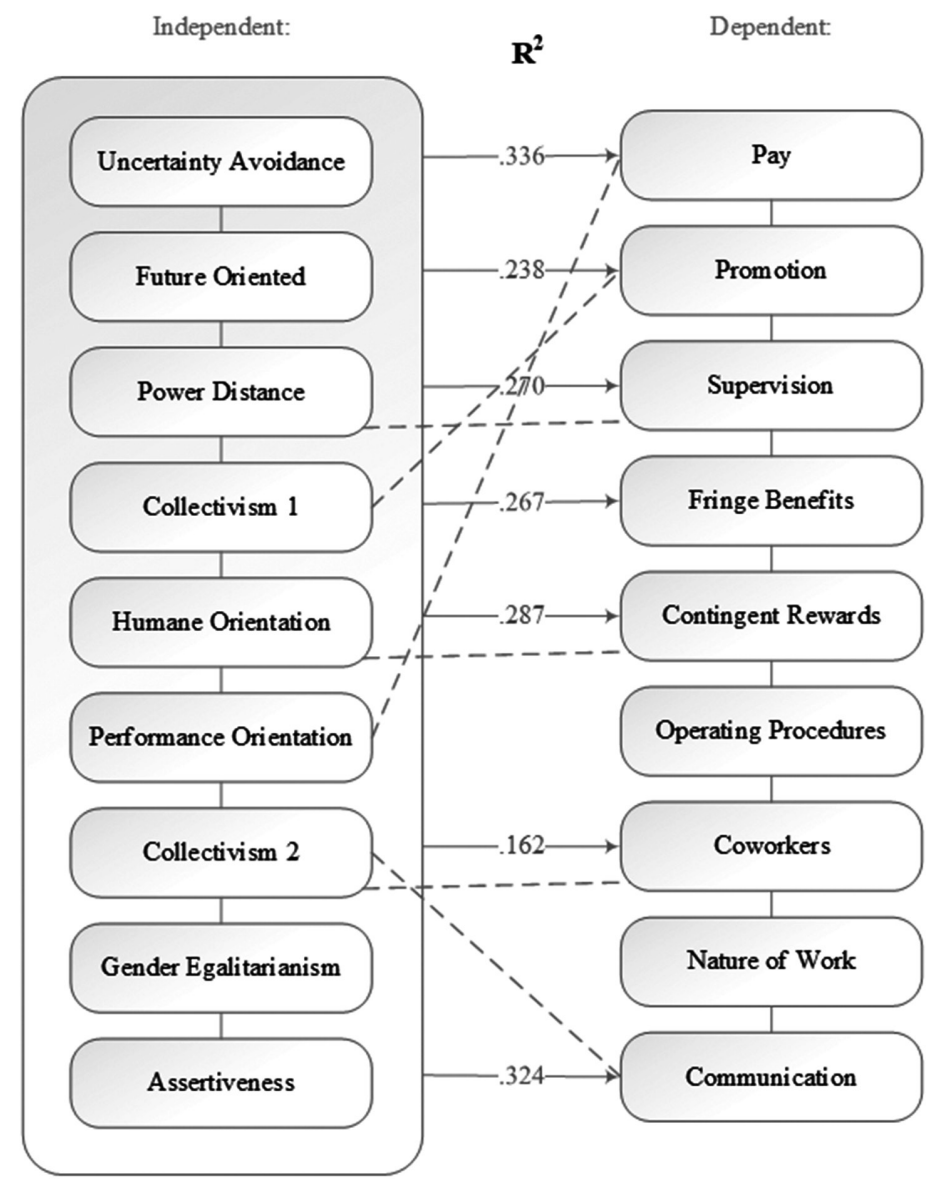

Figure 1. Multiple regression

Source: Authors calculation

Further results reveal that the correlation coefficient between national culture dimensions and the facet of job satisfaction in low Need for Achievement (NA) and high Need for Achievement sub-samples. In Table 7 we give the Pearson coefficients of the correlation.

Table 7. National culture dimensions and the facet of job satisfaction in low and high Need for Achievement sub-samples

\begin{tabular}{|c|c|c|c|c|c|c|c|c|c|c|}
\hline \multicolumn{2}{|c|}{ Need for Achievement } & NC1 & $\mathrm{NC2}$ & NC3 & NC4 & NC5 & NC6 & NC7 & NC8 & NC9 \\
\hline \multirow{9}{*}{ Low NA } & JS1 & $.300^{*}$ & $.353^{* *}$ & $-.360^{* *}$ & .075 & $.381^{* *}$ & $.408^{* *}$ & .085 & -.028 & -.172 \\
\hline & JS2 & .173 & $.318^{*}$ & -.148 & .061 & $.380^{* *}$ & .198 & .173 & -.058 & .015 \\
\hline & JS3 & $.295^{*}$ & $.381^{* *}$ & $-.430^{* *}$ & -.026 & $.426^{* *}$ & $.321^{*}$ & .145 & -.009 & -.171 \\
\hline & JS4 & $.299^{*}$ & .237 & -.159 & .017 & $.300^{*}$ & .122 & .213 & -.183 & .048 \\
\hline & JS5 & $.293^{*}$ & .189 & $-.343^{* *}$ & -.073 & $.429^{* *}$ & $.276^{*}$ & .027 & .119 & -.070 \\
\hline & JS6 & -.061 & -.059 & -.100 & .106 & $.300^{*}$ & .112 & -.241 & .140 & -.184 \\
\hline & JS7 & .155 & .053 & -.153 & -.088 & .234 & .143 & .050 & .088 & -.050 \\
\hline & JS8 & .145 & .145 & -.089 & -.128 & -.039 & .181 & .120 & .211 & .005 \\
\hline & JS9 & $.392^{* *}$ & .185 & $-.314^{*}$ & -.182 & $.354^{* *}$ & $.311^{*}$ & $.409^{* *}$ & .050 & .086 \\
\hline
\end{tabular}




\begin{tabular}{|c|c|c|c|c|c|c|c|c|c|c|}
\hline \multirow{9}{*}{ High NA } & JS1 & $.441^{* *}$ & $.425^{* *}$ & $-.400^{* *}$ & $.433^{* *}$ & $.300^{*}$ & $.589^{* *}$ & $.289^{*}$ & -.023 & .124 \\
\hline & JS2 & .211 & $.282^{*}$ & $-.486^{* *}$ & $.601^{* *}$ & .234 & $.407^{* *}$ & .141 & .132 & .041 \\
\hline & JS3 & $.335^{* *}$ & $.406^{* *}$ & $-.427^{* *}$ & $.469^{* *}$ & $.310^{*}$ & $.404^{* *}$ & .140 & .149 & .143 \\
\hline & JS4 & $.477^{* *}$ & $.489^{* *}$ & $-.357^{* *}$ & $.343^{* *}$ & $.294^{*}$ & $.629^{* *}$ & .254 & -.053 & .207 \\
\hline & JS5 & $.422^{* *}$ & $.420^{* *}$ & $-.382^{* *}$ & $.635^{* *}$ & $.435^{* *}$ & $.559^{* *}$ & $.314^{*}$ & .147 & .191 \\
\hline & JS6 & .016 & -.047 & .056 & .114 & -.104 & -.058 & .016 & .040 & -.059 \\
\hline & JS7 & $.300^{*}$ & $.410^{* *}$ & $-.268^{*}$ & $.550^{* *}$ & $.349^{* *}$ & $.376^{* *}$ & $.343^{* *}$ & .214 & .044 \\
\hline & JS8 & .240 & $.315^{*}$ & -.135 & $.314^{*}$ & $.301^{*}$ & $.302^{*}$ & $.324^{*}$ & .045 & .167 \\
\hline & JS9 & $.321^{*}$ & $.347^{* *}$ & $-.454^{* *}$ & $.498^{* *}$ & $.324^{*}$ & $.497^{* *}$ & $.323^{*}$ & .018 & .022 \\
\hline
\end{tabular}

** Correlation is significant at the 0.01 level (2-tailed).

* Correlation is significant at the 0.05 level (2-tailed).

Source: Authors calculation

In Table 8 we give the Pearson coefficients of the correlation between national culture dimensions and the facet of job satisfaction for gender, men and women sub-samples.

Table 8. National culture dimensions and the facet of job satisfaction for men and women sub-samples

\begin{tabular}{|c|c|c|c|c|c|c|c|c|c|c|}
\hline \multicolumn{2}{|l|}{ Gender } & $\mathrm{NC1}$ & $\mathrm{NC2}$ & NC3 & $\mathrm{NC4}$ & NC5 & NC6 & NC7 & NC8 & NC9 \\
\hline \multirow{9}{*}{$\begin{array}{l}\text { Men } \\
N=65\end{array}$} & JS1 & $.467^{* *}$ & $.469^{* *}$ & $-.412^{* *}$ & .233 & $.311^{*}$ & $.465^{* *}$ & .180 & -.084 & -.170 \\
\hline & JS2 & .131 & $.290^{*}$ & -.097 & $.384^{* *}$ & .228 & .159 & .196 & -.094 & -.097 \\
\hline & JS3 & $.328^{* *}$ & $.391^{* *}$ & $-.398^{* *}$ & .108 & $.337^{* *}$ & $.288^{*}$ & $.261^{*}$ & .012 & -.220 \\
\hline & JS4 & $.436^{* *}$ & $.315^{*}$ & -.143 & .064 & .221 & .226 & $.259^{*}$ & -.171 & .003 \\
\hline & JS5 & $.386^{* *}$ & $.287^{*}$ & -.216 & $.309^{*}$ & $.361^{* *}$ & $.266^{*}$ & .232 & .089 & -.070 \\
\hline & JS6 & -.103 & -.147 & -.076 & .144 & .113 & .029 & -.177 & .106 & -.180 \\
\hline & JS7 & .240 & .218 & -.093 & .195 & .168 & .162 & $.267^{*}$ & -.022 & -.083 \\
\hline & JS8 & .241 & .211 & .036 & .218 & .048 & .141 & $.349^{* *}$ & .063 & .063 \\
\hline & JS9 & $.368^{* *}$ & $.369^{* *}$ & -.214 & .186 & .193 & $.310^{*}$ & $.448^{* * *}$ & -.019 & .057 \\
\hline \multirow{9}{*}{$\begin{array}{l}\text { Women } \\
\mathrm{N}=55\end{array}$} & JS1 & $.272^{*}$ & .244 & $-.392^{* *}$ & $.309^{*}$ & $.334^{*}$ & $.514^{* *}$ & .005 & .120 & .190 \\
\hline & JS2 & .259 & $.276^{*}$ & $-.597^{* *}$ & $.344^{*}$ & $.355^{* *}$ & $.495^{* *}$ & .017 & .260 & .192 \\
\hline & JS3 & $.272^{*}$ & $.322^{*}$ & $-.449^{* *}$ & $.286^{*}$ & $.343^{*}$ & $.360^{* *}$ & -.038 & .210 & .244 \\
\hline & JS4 & $.358^{* *}$ & $.457^{* *}$ & $-.420^{* *}$ & $.318^{*}$ & $.371^{* *}$ & $.640^{* *}$ & .109 & .011 & $.316^{*}$ \\
\hline & JS5 & $.357^{* *}$ & $.326^{*}$ & $-.609^{* *}$ & $.315^{*}$ & $.507^{* *}$ & $.613^{* *}$ & -.035 & $.327^{*}$ & $.311^{*}$ \\
\hline & JS6 & .078 & .077 & .014 & .090 & .017 & -.012 & -.112 & .074 & -.055 \\
\hline & JS7 & .207 & .234 & $-.278^{*}$ & .242 & $.383^{* *}$ & $.298^{*}$ & .117 & $.373^{* *}$ & .097 \\
\hline & JS8 & .103 & .180 & -.225 & -.105 & .171 & .253 & .040 & .260 & .124 \\
\hline & JS9 & $.308^{*}$ & .116 & $-.532^{* *}$ & .188 & $.483^{* *}$ & $.519^{* *}$ & .246 & .115 & .066 \\
\hline
\end{tabular}

** Correlation is significant at the 0.01 level (2-tailed).

* Correlation is significant at the 0.05 level (2-tailed).

Source: Authors calculation

We used hierarchical regression analysis in order to investigate the moderating effect of Gender and Need for Achievement on the regression between dimensions of national culture (as an independent variable) and the facet of job satisfaction (as a dependent variable). The hierarchical regression analysis consists of three steps. In the first step, national culture is a predictor, in the second step national culture and moderator (Gender or Need for Achievement) are predictors, and in the last step, an interactive variable is added into the model. The moderating effect was tested by examining the change in the R-squared attributable to the interaction term. If the interaction term added at the final stage of the regression analysis produced a significantly increased amount of variance explained in the outcome variable (R-squared), we could say that the moderator effect occurs between national culture dimensions and facet of job satisfaction. 
Table 9 shows the results of the examination of the moderator effect of Need for Achievement on the influence of dimensions of national culture and facet of job satisfaction. In Table 9, only the results related to R- square and F-change statistics that support $\mathrm{H} 3$ are presented.

Table 9. Hierarchical regression analysis (R square and F-change) for Need for Achievement

\begin{tabular}{|c|c|c|c|c|c|c|c|c|c|c|}
\hline \multirow{2}{*}{\multicolumn{2}{|c|}{$\begin{array}{c}\text { Independent } \\
\text { variables }\end{array}$}} & \multicolumn{9}{|c|}{ Dependent variables } \\
\hline & & JS1 & JS2 & JS3 & JS4 & JS5 & JS6 & JS7 & JS8 & JS9 \\
\hline 1 & $\begin{array}{l}\text { NC2 } \\
\text { R2 } \\
\text { F ch } \\
\text { NA } \\
\text { R2 } \\
\text { F ch } \\
\text { Interac_NA } \\
\text { R2 } \\
\text { F ch } \\
\end{array}$ & & & & & $\begin{array}{l}.106 \\
14.035^{* *} \\
.107 \\
.053 \\
.139 \\
4.403^{*} \\
\end{array}$ & & $\begin{array}{l}.057 \\
7.114^{* *} \\
.103 \\
6.028^{*} \\
.140 \\
5.014 * \\
\end{array}$ & & \\
\hline 3 & \begin{tabular}{|l}
$\mathrm{NC} 3$ \\
$\mathrm{R} 2$ \\
F ch \\
$\mathrm{NA}$ \\
$\mathrm{R} 2$ \\
F ch \\
Interac_NA \\
R2 \\
F ch \\
\end{tabular} & & $\begin{array}{l}.108 \\
14.263^{* *} \\
\\
.116 \\
1.057 \\
.159 \\
6.016^{*}\end{array}$ & & & & & & & \\
\hline 1 & $\begin{array}{l}\text { NC4 } \\
\text { R2 } \\
\text { F ch } \\
\text { NA } \\
\text { R2 } \\
\text { F ch } \\
\text { Interac_NA } \\
\text { R2 } \\
\text { F ch }\end{array}$ & $\begin{array}{l}.084 \\
10.781 * * \\
.098 \\
1.801 \\
\\
.132 \\
4.559 *\end{array}$ & $\begin{array}{l}.139 \\
19.024 * * \\
\\
.142 \\
.429 \\
.219 \\
11.358 * *\end{array}$ & $\begin{array}{l}.041 \\
5.085^{*} \\
\\
.049 \\
.955 \\
\\
.100 \\
6.551^{*}\end{array}$ & & $\begin{array}{l}.106 \\
13.935^{* *} \\
.106 \\
.001 \\
.235 \\
19.675^{* *}\end{array}$ & & $\begin{array}{l}.055 \\
6.877^{* *} \\
\\
.096 \\
5.231^{*} \\
\\
.203 \\
15.716^{* *}\end{array}$ & $\begin{array}{l}.005 \\
.579 \\
\\
.055 \\
6.251^{*} \\
\\
.140 \\
11.370^{* *}\end{array}$ & $\begin{array}{l}.036 \\
4.383^{*} \\
.055 \\
2.349 \\
\\
.183 \\
18.244^{* *}\end{array}$ \\
\hline 1 & \begin{tabular}{|l|} 
NC5 \\
R2 \\
F ch \\
NA \\
R2 \\
F ch \\
Interac_NA \\
R2 \\
F ch \\
\end{tabular} & & & & & & $\begin{array}{l}.007 \\
.834 \\
\\
.035 \\
3.401 \\
\\
.075 \\
5.019 *\end{array}$ & & & \\
\hline 3 & $\begin{array}{l}\text { NC6 } \\
\text { R2 } \\
\text { F ch } \\
\text { NA } \\
\text { R2 } \\
\text { F ch } \\
\text { Interac_NA } \\
\text { R2 } \\
\text { F ch }\end{array}$ & & & & $\begin{array}{l}.171 \\
24.423^{* *} \\
.172 \\
.040 \\
.220 \\
7.235^{* *}\end{array}$ & & & & & \\
\hline
\end{tabular}

Source: Authors calculation

Need for Achievement is the moderator of relations between NC2 (Future Orientation) and JS5 (Contingent Rewards) and JS7 (Coworkers); NC3 (Power Distance) and JS2 (Promotion); NC4 (Collectivism 1) and JS1 (Pay), JS2 (Promotion), JS3 (Supervision), JS5 (Contingent Rewards), JS7 (Coworkers), JS8 (Nature of Work) and JS9 (Communication); NC5 (Humane Orientation) 
and JS6 (Operating Procedures); NC6 (Performance Orientation) and JS4 (Fringe Benefits) in the tourism service providers in Serbia.

The results of the examination of the moderator effect of respondents' gender on the influence of dimensions of national culture and facet of job satisfaction are shown in Table 10. In Table 10 , only the results related to R-square and F-change statistics that support H4 are presented.

Table 10. Hierarchical regression analysis (R square and F-change) for Gender

\begin{tabular}{|c|c|c|c|c|c|c|c|c|c|c|}
\hline \multirow{2}{*}{\multicolumn{2}{|c|}{$\begin{array}{c}\text { Independent } \\
\text { variables }\end{array}$}} & \multicolumn{9}{|c|}{ Dependent variables } \\
\hline & & JS1 & JS2 & JS3 & JS4 & JS5 & JS6 & JS7 & JS8 & JS9 \\
\hline 1 & \begin{tabular}{|l}
$\mathrm{NC2} 2$ \\
$\mathrm{R} 2$ \\
$\mathrm{~F}$ ch \\
$\mathrm{NA}$ \\
$\mathrm{R} 2$ \\
$\mathrm{~F}$ ch
\end{tabular} & & $\begin{array}{l}.108 \\
14.263^{* *} \\
.154 \\
6.343^{*}\end{array}$ & & & & & & & \\
\hline 1 & \begin{tabular}{|l|}
$\mathrm{NC} 2$ \\
$\mathrm{R} 2$ \\
$\mathrm{~F}$ ch \\
$\mathrm{NA}$ \\
$\mathrm{R} 2$ \\
$\mathrm{~F}$ ch \\
\end{tabular} & & $\begin{array}{l}.107 \\
14.086^{* *} \\
.137 \\
4.105^{*} \\
\end{array}$ & & \begin{tabular}{|l}
.171 \\
$24.423 * *$ \\
\\
.206 \\
$5.060^{*}$ \\
\end{tabular} & & & & & \\
\hline 1 & \begin{tabular}{|l|}
$\mathrm{NC} 2$ \\
$\mathrm{R} 2$ \\
$\mathrm{~F}$ ch \\
$\mathrm{NA}$ \\
$\mathrm{R} 2$ \\
$\mathrm{~F}$ ch \\
\end{tabular} & & & & & & & \begin{tabular}{|l}
.025 \\
3.046 \\
\\
.065 \\
$4.940^{*}$
\end{tabular} & & \\
\hline
\end{tabular}

Source: Authors calculation

Gender is the moderator of relations between NC3 (Power Distance) and JS2 (Promotion); NC6 (Performance Orientation) and JS2 (Promotion) and JS4 (Fringe Benefits); NC8 (Gender Egalitarianism) and JS7 (Coworkers) in the tourism service providers in Serbia.

\section{DISCUSSION}

The main goal of this research was to investigate the contribution of all the independent variables, dimensions of National Culture, to the total R-square in the regression with the aspect of Job Satisfaction as a dependent variable, and the moderating effect of Gender and Need for Achievement in the relation between National Culture and Job Satisfaction in the tourism service providers in Serbia.The results obtained confirm that dimensions of national culture are predictors of job satisfaction (Hypothesis One). Also, the results confirm that the Need for Achievement (Hypothesis Two) and Gender (Hypothesis Three) are moderators on the relations between National Culture dimensions and the aspect of Job Satisfaction in the tourism service providers in Serbia. These findings are discussed in more detail below.

\subsection{National culture as a predictor of job satisfaction in tourism service providers}

GLOBE national culture is a significant predictor of all facets of job satisfaction except Operating Procedures (JS6) and Nature of Work (JS8). Pay and remuneration satisfaction is in direct proportion to the degree to which a collective encourages and rewards tourism service provider employees for performance improvement and high standards. The greatest influence on promotion opportu- 
nities satisfaction has the degree to which organizational and societal institutions encourage and reward tourism provider employees' integration into groups and collective actions. The greatest influence on immediate supervisor satisfaction has the degree to which tourism provider employees accept an unequal distribution of power and status privileges. The greatest influence on satisfaction with appreciation, recognition, and rewards have the degree to which a collective encourages and rewards tourism provider employees or fairness, kindness, generosity, and altruism. The greatest influence on co-workers' satisfaction and job tasks themselves have the degree to which tourism provider employees express pride, loyalty, and cohesiveness in their organizations or families.

\subsection{Uncertainty avoidance}

In the general sample "as is" value of Uncertainty Avoidance (NC1) is 3.80, and "should be" is 5.60. Taking into consideration the values of Overall GLOBE Data, tourism provider employees' values are similar to "as is" lowest score and "should be" highest score. Tourism provider employees strive to a higher level of uncertainty avoidance, as part of national culture. It can be concluded that there is a strong tendency towards a system that is more orderly and system-oriented towards planning and moderate risks. On the other hand, this situation is probably a consequence of a long period of socialism. It is possible that for this reason employees rely on the past, the period when there was a sense of security, orientation towards rules, and social order (Grachev et al., 2007). Uncertainty avoidance has statistically positive and significant correlations with all facets of job satisfaction, except Operating Procedures (JS6). Hence, the increase of the perception of Uncertainty Avoidance is accompanied by the increase of satisfaction with all aspects of the job, except satisfaction with operating policies and procedures.

\subsection{Future orientation}

In the general sample "as is" value of Future Orientation (NC2) is 2.28, and the "should be" value is 6.23. Based on Overall GLOBE Data, tourism provider employees" value "as is" is lower than the Overall lowest score and the "should be" value is similar to the highest Overall score. In the general sample, Future Orientation has the lowest value of all national culture dimensions. The future orientation dimension is a reflection of the internally consistent set of practices and values that influence the way the society defines success and adapts to external challenges and the way the society manages relationships with its people (Jesuino, 2002). Weaker Future Orientation societies value instant gratification and place higher priorities on immediate rewards. Organizations have a shorter strategic orientation, emphasize leadership that focuses on repetition of reproducible and routine sequences (House et al., 2004), and that influence intrapreneurship. Accordingly, reflections of tourism provider employees on the desired state in the future are reflected in planning, visionary leadership, long-term success, organizations with a long-term strategic orientation, collective safety nets (House et al., 2002; House et al., 2004). The significant difference between "as is" and "should be" values indicates that tourism provider employees do not expect fast results, but they are ready for change and investments for future payoffs over immediate consumption. Future Orientation has statistically significant and positive correlations with all facets of job satisfaction, except Operating Procedures (JS6). The increase of the perception of Future Orientation is accompanied by the increase of satisfaction with all aspects of the job, except satisfaction with operating policies and procedures.

We obtained that the Need for Achievement moderates the relation between Future Orientation and Contingent Rewards (JS5) and Coworkers (JS7). In the high Need for Achievement sub-sample, 
relations between Future Orientation and Contingent Rewards (.420**) and Coworkers (.410**) are significant and positive and in the low sub-sample coefficients are positive, but not significant. A high value of the Need for Achievement can help managers in the tourism service providers in Serbia to be more motivated to face the challenges and to learn, which may help them to achieve success. Also, it can encourage tourism provider employees to perform future-oriented behaviors such as planning and delaying gratification. High achiever tourism provider employees pursue opportunities to create long term competitive advantages that enable intrapreneurship, appreciation and recognition from coworkers and supervisors, and rewards, as a measurement for accomplishments.

\subsection{Power Distance}

In the general sample "as is" value of Power Distance (NC3) is 6.16, and the "should be" value is 2.98. Based on Overall GLOBE Data, tourism provider employees' value "as is" is higher than the Overall highest score and the "should be" value is similar to the lowest Overall score. In the general sample, Power Distance has the highest value of all National Culture dimensions. Hofstede (1981; 2001) first identified a high-power distance in Serbia. Serbia, and other countries with a high value on power distance, are characterized by social, economic, and political stratification, and those in power expect and accept obedience (Javidan et al. 2006). Societies with high Power Distance have a large middle class and higher public corruption, do not ensure equal opportunities and the power is seen as a source of corruption, coercion, and dominance (House et al., 2004). Power Distance has statistically significant and negative relations with all facets of job satisfaction, except Operating Procedures (JS6) and Nature of Work (JS8) where coefficients are negative, but they are not significant. The increase of the perception of Power Distance is accompanied by the decrease of satisfaction with all aspects of the job, except satisfaction with operating policies and procedures and satisfaction with job tasks themselves.

Multiple regression shows that Need for Achievement is a moderator of relations between Power Distance (NC3) and Promotion (JS2). In the high Need for Achievement sub-sample, the coefficient between Power Distance and Promotion (-.486**) is negative and significant and in the low sub-sample coefficient is negative, but not significant. In the high value Need for Achievement sub-sample, the increase of the perception of power distance is followed by a faster decrease of satisfaction with promotion opportunities than in the low Need for Achievement sub-sample. In tourism service provider in Serbia, especially in the utility companies (state owned), promotion opportunities partly depend on personal connections and political authorities in the broader community. This practice has a long tradition in Serbian organizations since the period of socialism. High achiever tourism provider employees prefer conditions that will enable their need to face challenges, to learn constantly and to succeed as a measurement for accomplishments, and as a result, they will be more satisfied with equal promotion opportunities.

Multiple regression shows that Gender is a moderator of relations between Power Distance and Promotion (JS2). In the Female sub-sample, the correlation coefficient is negative and significant $\left(-.597^{* *}\right)$, and in the Male sub-sample coefficient is negative but not significant (-.097). Promotion satisfaction of women tourism provider employees depends on equal promotion opportunities. In Serbia, men are more involved in politics and probably have/rely more on social connections than women. It could be assumed that men in Serbia value social and political connections and even support such a grounded system that affects social status and prestige; unlike women who do not support inequalities and believe that promotion opportunities should depend on results and achievements. 


\subsection{Collectivism 1}

In the general sample "as is" value of Collectivism 1 (NC4) is 3.64, and the "should be" value is 4.88. Taking into consideration the values of Overall GLOBE Data, the values of tourism provider employees are similar to "as is" lowest score and "should be" highest score. Serbia is considered to be a collective society, but values "as is" show differently. Based on "should be" tourism provider employees' traditional institutional values are still important (which have roots in socialism). In their opinion, goals and group interest "should be" over individual goals and interests and important decisions ,should be" made by the group (Javidan, House, 2001). Collectivism 1 has statistically significant and positive correlations with all facets of job satisfaction, except Fringe Benefits (JS4) and Operating Procedures (JS6) where coefficients are not significant. The increase of the perception of Collectivism 1 is accompanied by the increase of satisfaction with all aspects of the job, except satisfaction with monetary and nonmonetary fringe benefits and operating policies in tourism provider employees.

Multiple regression shows that Need for Achievement is a moderator of relations between Collectivism 1 and Pay (JS1), Promotion (JS2), Supervision (JS3), Contingent Rewards (JS5), Coworkers (JS7), Gender Egalitarianism (NC8), Assertiveness (NC9). In the high value Need for Achievement sub-sample, the increase of the perception of institutional collectivism is followed by a faster increase of satisfaction with Pay, Promotion, Supervision, Contingent Rewards, Coworkers, Gender Egalitarianism, and Assertiveness than in the low Need for Achievement sub-sample. Under the increase of institutional collectivism, high achieve tourism provider employees feel the responsibility to achieve good relationships and communication with supervisors and all coworkers with modesty and tenderness. They tend to promote intrapreneurship to achieve opportunities and rewards as an organization. Their expectations of pay are related to the Serbian economy.

\subsection{Humane Orientation}

In the general sample "as is" value of Humane Orientation (NC5) is 3.29, and the "should be" value is 6.28. The "as is" value is similar to the lowest Overall GLOBE Data and the "should be" value is higher than Overall highest score. Kabasakal \& Bodur (2004) indicate that social culture based on bureaucratic practice, formal relationships, and formalized procedures is typical in cultures with low human orientation. According to the opinion of tourism service provider employees, the well-being of others "should be" individual responsibility, and has high value towards altruism, kindness, love, and generosity in society (Triandis, 1995). Humane Orientation has statistically significant and positive correlations with all facets of job satisfaction, except Operating Procedures (JS6) and Nature of Work (JS8) where coefficients are positive but not significant. The increase of the perception of Humane Orientation is accompanied by the increase of satisfaction with all aspects of the job, except satisfaction with operating policies and job tasks in tourism provider employees in Serbia.

Multiple regression shows that Need for Achievement is a moderator of relations between Humane Orientation and Operating Procedures (JS6). In the low value Need for Achievement sub-sample, the increase of the perception of Humane Orientation is followed by a faster increase of satisfaction in tourism provider employees. In the high Need for Achievement, the sub-sample relation is negative and not significant. The increase of Humane Orientation in low achieve tourism provider employees is accompanied by the increase of satisfaction with Operating Procedures. These results can be explained based on high achievers' ambition, goal-orientation, task-orien- 
tation, and tendency to succeed in a competitive environment. In relation to a set of standards, they tend to overachieve and push limits. At some point that can mean they are less human-oriented. Required rules and procedures can affect intrapreneurship. Also, they want to compete with others, more than to help others to achieve. In case of low need for achievement, employees are less interested in the activities that provide high skill and challenge at work and try to avoid competence assessment (Eisenberger et al., 2005). That makes them more human-oriented and ready to help others and to receive help if needed. Required rules and procedures ensure security, and predictability, give them a sense of stability and that affects their satisfaction.

\subsection{Performance Orientation}

In the general sample "as is" value of Performance Orientation (NC6) is 3.07, and the "should be" value is 6.52. "As is" value is higher than lowest Overall GLOBE Data and "should be" value is lower than Overall highest score. Tourism service provider employees think that collective ,should" encourage more and reward members for performance improvement. Based on the current situation, social and family relations and seniority and experience are more important than results, education and learning and taking initiative (House et al. 2004). Performance Orientation has statistically significant and positive correlations with all facets of job satisfaction, except Operating Procedures (JS6). The strongest relation is with Pay (JS1, 0.525**) and the weakest with Nature of Work (JS8, 0.212*).

Multiple regression shows that Need for Achievement is a moderator of relations between Performance Orientation and Fringe Benefits (JS4). In the high Need for Achievement sub-sample, the relation between Performance Orientation and Fringe Benefits $\left(.629^{* *}\right)$ is positive and significant and in the low sub-sample coefficient is positive, but not significant (.121). In the high value Need for Achievement sub-sample, the increase of the perception of Performance Orientation is followed by a faster increase of satisfaction with monetary and nonmonetary fringe benefits. High achievement tourism provider employees expect that organization invests in training and education, to promote performance improvements and intrapreneurship. They are more satisfied in organizations that give performance feedback and reward performance with monetary and nonmonetary fringe benefits.

Multiple regression shows that Gender is a moderator of relations between Performance Orientation and Promotion (JS2) and Fringe Benefits (JS4). In the female sub-sample, Performance Orientation is followed by a faster increase of satisfaction with promotion opportunities and monetary and nonmonetary fringe benefits. In the male sub-sample, the relation is positive, but not statistically significant. Also, in the male sub-sample the value of culture dimension Performance Orientation (3.39) is higher than in the female sub-sample (2.69). In this case, encouragement and rewards for performance improvement do not affect male satisfaction. That could mean that they simply do as best as they can, in any conditions. On the other hand, females are ready to improve their performance. If the organization encourages and rewards results, they expect promotion opportunities and fringe benefits for their efforts. A tourism service provider organization that rewards performance has more satisfied female employees.

\subsection{Gender Egalitarianism}

In the general sample "as is" value of Gender Egalitarianism (NC8) is 3.37, and the "should be" value is 4.97. "As is" value is between lowest and highest Overall GLOBE Data and "should be" value is lower than Overall highest score. Tourism service provider employees value equal opportunities for all and more women in positions of authority. 
Multiple regression shows that Gender is a moderator of relations between Gender Egalitarianism and Coworkers (JS7). In the female sub-sample this relation is positive and significant $\left(.373^{* *}\right)$ and in the male sub-sample, the relation is negative and not significant (-.022). In the female sub-sample increased perception of Gender Egalitarianism is followed by increased satisfaction with Coworkers. Women in tourism service provider organizations, where is a lack of gender barriers, feel safer and more satisfied with the people they work with.

\section{CONCLUSION}

This paper has investigated the influence of national culture on the facets of job satisfaction in tourism service provider managers. Our results provide empirical evidence that GLOBE national culture is a predictor of all facets of job satisfaction except Operating Procedures and Nature of Work. National culture dimensions influence all facets of job satisfaction except Operating Procedures. In the general sample, dimensions of national culture that do not influence the facets of job satisfaction are Gender Egalitarianism and Assertiveness. GLOBE national culture value "should be" is an important factor that influences the job satisfaction. Results also prove that the Need for Achievement is a moderator of some relations between the national culture dimension as an independent variable and job satisfaction as a dependent variable. Also, high value of Need for Achievement increases the level of satisfaction with many facets of the job and it is very important for intrapreneurship development. Low value of Need for Achievement increases the level of satisfaction only with Operating Procedures. We prove that Gender is a moderator of some relations between the national culture dimension as an independent variable and job satisfaction as a dependent variable. Women in tourism service provider organizations have a higher level of satisfaction with facets of the job.

In modern conditions of constant changes, the increased need for the development of the service sector and destination competitiveness, an important factor in the quality of the tourist destination and one of the main destination stakeholders are tourism providers. National culture is especially important for intrapreneurship in the tourism industry, particularly for tourism providers, in order to maximize the quality of the tourist destination. This paper, among other things, provides insight into the possibilities and conditions for the development of intrapreneurship in the tourism sector providers. Besides that, managers should take into consideration that there are no applicable actions to eliminate national culture influence on the job satisfaction. The influence could be managed to some extent with organizational culture.

\section{REFERENCES}

Alajmi, S., Dennis, C., \& Altayab, Y. (2011). The effect of national culture on service provision within Takaful industry: A comparative study in Kuwait and Egypt. Journal of Islamic Marketing, 2(3), 225-245. https://doi.org/10.1108/17590831111164769

Auer Antoncic, J., \& Antoncic, B. (2018). Need for achievement of the entrepreneur, intrapreneurship, and the growth of companies in tourism and trade. 3rd International Thematic Monograph: Modern Management Tools and Economy of Tourism Sector in Present Era, 259-267. https://doi.org/10.31410/tmt.2018.259

Bednarska, M.A. \& Szczyt, M. (2015). Variations in job satisfaction in service industries: comparative international analysis. Foresight, 17(6), 599-615. https://doi.org/10.1108/FS-08-2013-0037

Blyton, P. (2001). The General and the Particular in Cross-National Comparative Research. Applied Psychology: An International Review. Vol. 50(4), 590-595. https://doi.org/10.1111/14640597.00074 
Díaz-Carrión, R., Navajas-Romero, V., \& Casas-Rosal, J.C. (2020). Comparing working conditions and job satisfaction in hospitality workers across Europe. International Journal of Hospitality Management, 90, 102631. https://doi.org/10.1016/j.ijhm.2020.102631.

Dickson, M. W., Aditya, R. N., \& Chhokar, J. S. (2000). Definition and Interpretation in Cross-cultural Organizational Culture Research: Some Pointers from the GLOBE Research Program. In N.M Ashkanasy, C.P.M Wilderom, \& M.F Peterson (Eds.), Handbook of organizational culture and climate (pp. 447-465). Thousand Oaks, CA: Sage Publications, Inc.

Dickson, M.W., Den Hartog, D.N., \& Mitchelson, J.K. (2003). Research on leadership in a cross-cultural context: Making progress and raising new questions. The Leadership Quarterly, 14(6), 729-768. https://doi.org/10.1016/j.leaqua.2003.09.002

Dimitratos, P., Petrou, A., Plakoyiannaki, E., \& Johnson, J. E. (2011). Strategic decision-making processes in internationalization: Does national culture of the focal firm matter? Journal of World Business, 46(2), 194-204. https://doi.org/10.1016/j.jwb.2010.05.002

Eisenberger, R., Jones, J. R., Stinglhamber, F., Shanock, L., \& Randall, A. T. (2005). Flow experiences at work: For high need achievers alone? Journal of Organizational Behavior, 26(7), 755-775. https://doi.org/10.1002/job.337

Fargher, S., Kesting, S., Lange, T., \& Pacheco, G. (2008): Cultural heritage and job satisfaction in Eastern and Western Europe. International Journal of Manpower, 29, 630-650. https:/doi. org $/ 10.1108 / 01437720810908938$

Feichtinger, C., \& Fink, G. (1998). The collective culture shock in transition countries theoretical and empirical implications. Leadership and Organization Development Journal, 19(6), 302308. https://doi.org/10.1108/01437739810240821

Fink, G., \& Holden, N. (2002). Collective culture shock. Contrastive reactions to radical systemic change. EI Working Papers, Europainstitut, 45. Forschungsinstitut für Europafragen, WU Vienna University of Economics and Business, Vienna.

Finkelstein, M.A. (2011). Correlates of individualism and collectivism: Predicting volunteer activity. Social Behavior and Personality: An International Journal, 39(5), 597-606. https://doi. org/10.2224/sbp.2011.39.5.597

Finogenow, M. (2017). Need for Achievement. In: Zeigler-Hill V., Shackelford T. (eds) Encyclopedia of Personality and Individual Differences. Springer, Cham. https://doi.org/10.1007/978-3-31928099-8 537-1

Gerhart, B. (2008). Cross Cultural Management Research: Assumptions, Evidence, and Suggested Directions. International Journal of Cross-Cultural Management, 8(3), 259-274. https://doi. org/10.1177/1470595808096669

Grachev, M., Rogovsky, N., \& Rakitski, B. (2007). Business Leadership and Culture in Transitional Economy: A Case of Russia. In: J. Chhokar, F. Brodbeck, and R. House (eds.), Culture and Leadership Across the World: the GLOBE Book of In-Depth Studies of 25 Societies. Mahwah, NJ: Lawrence Erlbraun, 803-831. https://doi.org/10.4324/9780203936665

Hall, E.T. (1976). Beyond Culture. New York, N.Y. Anchor Press-Doubleday. ISBN 0385124740

Hofstede, G. (1980). Culture's Consequences - International Differences in Work-Related Values. Abridged Edition, Newbury Park, CA: Sage. ISBN: 9780803913066

Hofstede, G. (2001). Culture's Consequences, Comparing Values, Behaviours, Institutions, and Organizations Across Nations. Thousand Oaks, CA: Sage Publications. ISBN: 9780803973244

Hofstede, G.H., Hofstede, G.J., \& Minkov, M. (2010). Cultures and organizations: software of the mind. Maidenhead: McGraw-Hill. ISBN: 100071664181

House R., Javidan M., Hanges P., \& Dorfman P. (2002). Understanding cultures and implicit leadership theories across the globe: an introduction to project GLOBE. Journal of Word Business, 37(1), 3-10. https://doi.org/10.1016/S1090-9516(01)00069-4 
House, R.J., Hanges, P.J., Javidan, M., Dorfman, P.W., \& Gupta. V. (2004). Leadership, culture, and organizations: The GLOBE study of 62 societies. Thousand Oaks, CA: Sage. ISBN: 9780761924012

House, R.J., Hanges, P.J., Javidan, M., Dorfman, P.W., \& Gupta. V. (2004): Leadership, culture, and organizations: The GLOBE study of 62 societies. Thousand Oaks, CA: Sage. ISBN: 9780761924012

House, R.J., Hanges, P.J., Ruiz-Quintanilla, S.A., Dorfman, P.W., Falkus, S.A., \& Ashkanasy, N.M. (1999). Cultural influences on leadership and organizations: Project Globe. In W.H. Mobley, M.J. Gessner \& V. Arnold (Ed.), Advances in Global Leadership (2nd Ed.) (pp. 171-233). Bingley, UK: Emerald Group Publishing Ltd.

Hui, M., Au, K., \& Fock, H. (2004). Empowerment effects across cultures. Journal of International Business Studies, 35, 46-60. https://doi.org/10.1057/palgrave.jibs. 8400067

Hwang, I.S., \& Chi, D.J. (2005). Relationships among internal marketing, employee job satisfaction and international hotel performance: An empirical study. International Journal of Management, 22(2), 285-293.

Javidan, M., \& House, R.J. (2001): Cultural Acumen for the Global Manager: Lessons from Project GLOBE. Organizational Dynamics. 29(4), 289-305. https://doi.org/10.1016/S00902616(01)00034-1

Javidan, M., Dorfman, P.W., Sully de Luque, M. F., \& House, R.J. (2006). In the eye of the beholder: cross cultural lessons in leadership from project GLOBE. Academy of Management Perspectives, 20(1), 67-90. https://doi.org/10.5465/amp.2006.19873410

Jesuino, J.C. (2002). Latin Europe cluster: from South to North. Journal of World Business, 37(1), 81-89. https://doi.org/10.1016/S1090-9516(01)00076-1

Judge, T.A., Thoresen, C.J., Bono, J.E., \& Patton, G.K. (2001). The job satisfaction-job performance relationship: A qualitative and quantitative review. Psychological Bulletin, 127(3), 376-407. https://oi.org/10.1037/0033-2909.127.3.376

Kabasakal, H., \& Bodur, M. (2004). Humane Orientation in Societies, Organizations, and Leader Attributes. In Culture, Leadership and Organizations, The GLOBE Study of 62 Societies, eds. R.J. House, P.J. Hanges, M. Javidan, P.W. Dorfman and V. Gupta, Thousand Oaks, CA: Sage, 564-601. ISBN: 9780761924012

Kirin, S., \& Gavrić, G. (2017). Improvement of organizational culture as a managing tool for achieving business success in Tourism-case study for Serbia. Second International Thematic Monograph - Thematic Proceedings: Modern Management Tools and Economy of Tourism Sector in Present Era, 302-317. ISBN 978-86-80194-10-3

Kluckhohn, F.R., \& Strodtbeck, F.L. (1961). Variations in value orientations. Oxford, UK: Row, Peterson.

Locke, E.A. (1976). The nature and causes of job satisfaction. In M. D. Dunnette (Ed.), Handbook of industrial and organizational psychology, 1297-1349. Chicago: Rand-McNally.

Mason, P., \& Cheyne, J. (2000). Residents Attitudes to Proposed Tourism Development. Annals of Tourism Research, 27(2), 391 - 411. https://doi.org/10.1016/S0160-7383(99)00084-5

McClelland, D.C. (1961). The achieving society. Princeton, NJ: Van Nostrand. ISBN 0029205107

McClelland, D.C. (1985). Human motivation. Glenview, IL: Scott, Foresman. ISBN 0673157911

Mebrahtu, T., Crossley, M.W., \& Johnson, D.F. (2000). Educational Development and Social Transformation in a Global Economy. In M., T. Crossley, \& D. M Johnson (Eds.), Globalisation, Educational Transformation and Societies in Transition, 9 - 20. Symposium Books. http://www. symposium-books.co.uk/books/bookdetails.asp?bid=35

Merritt, A. (2000). Culture in the Cockpit: do Hofstede's Dimensions Replicate? Journal of Cross-Cultural Psychology, 31(3), 283-301. https://doi.org/10.1177/0022022100031003001 
Morris, M., Davis, D., \& Allen, J. (1994). Fostering Corporate Entrepreneurship: Cross-Cultural Comparisons of the Importance of Individualism Versus Collectivism. Journal of International Business Studies 25, 65-89. https://doi.org/10.1057/palgrave.jibs. 8490849

Nazarian, A., Atkinson, P., \& Foroudi, P. (2017). Influence of national culture and balanced organizational culture on the hotel industry's performance. International Journal of Hospitality Management, 63, 22-32. https://doi.org/10.1016/j.ijhm.2017.01.003

Nedeljković Knežević, M., \& Pavluković, A. (2020). The Influence of GLOBE Culture Dimensions on Entrepreneurial Orientation in Tourism and Medical Service Sectors in Serbia. Geographica Pannonica, 24(2), 157-167. doi: 10.5937/gp24-24303

Paunković, Dž., \& Jovanović, V. (2016). National culture dimensions framework as managerial tool for business success in tourism. International Thematic Monograph - Thematic Proceedings: Modern Management Tools and Economy of Tourism Sector in Present Era, 317-336. ISBN 978-86-80194-04-2

Pavluković, V., Armenski, T., \& Alcántara-Pilar, J. M. (2017). Social impacts of music festivals: Does culture impact locals' attitude toward events in Serbia and Hungary? Tourism Management, 63, 42-53. https://doi.org/10.1016/j.tourman.2017.06.006

Sempane, M.E., Rieger, H.S., \& Roodt, G. (2002): Job Satisfaction In Relation To Organisational Culture. SA Journal of Industrial Psychology. 28(2), 23-30. https://doi.org/10.4102/sajip.v28i2.49

Spector, P.E. (1997): Job satisfaction: Application, assessment, cause, and consequences. Thousand Oaks, CA: Sage Publications, Inc. ISBN:9781452231549

Tiessen, J.H. (1997). Individualism, collectivism, and entrepreneurship: A framework for international comparative research. Journal of Business Venturing, 12(5), 367-384. https://doi. org/10.1016/S0883-9026(97)81199-8

Triandis, H. (1995). Individualism And Collectivism. New York: Routledge, https://doi. org/10.4324/9780429499845

Trompenaars, F., \& Hampden-Turner, C. (1997). Riding the waves of culture: understanding cultural diversity in business (2nd Edition). London, UK: Nicholas Brealey. ISBN: 0-7863-1125-8

Venaik S., \& Brewer, P.A. (2008). Contradictions in national culture: Hofstede vs GLOBE, in: Cantwell, J., \& Kiyak, T. (2008). Proceedings of the 50th Annual Meeting of the Academy of International Business. 50th Annual Meeting of the Academy of International Business (AIB), Milan, Italy. 274-274. 20 June - 3 July.

Vukonjanski J., Nikolić M., Hadžić O., Terek E., Nedeljković M. (2012): Relationship between GLOBE organizational culture dimensions, job satisfaction and leader-member exchange in Serbian organizations. Journal for East European Management Studies 17 (3), 333-368. ISSN 0949-6181. https://doi.org/10.5771/0949-6181-2012-3-333

Wakkee, I., Elfring, T., \& Monaghan, S. (2010). Creating entrepreneurial employees in traditional service sectors. International Entrepreneurship and Management Journal, 6, 1-21. https://doi. org/10.1007/s11365-008-0078-z

Wu, S., Matthews, L., \& Dagher, G. K. (2007). Need for achievement, business goals, and entrepreneurial persistence. Management Research News, 30(12), 928-941. https://doi. org $/ 10.1108 / 01409170710833358$

Zouni, G., \& Kouremenos, A. (2008). Do Tourism Providers Know their Visitors? An Investigation of Tourism Experience at a Destination. Tourism and Hospitality Research, 8(4), 282-297. https:// doi.org/10.1057/thr.2008.30 
